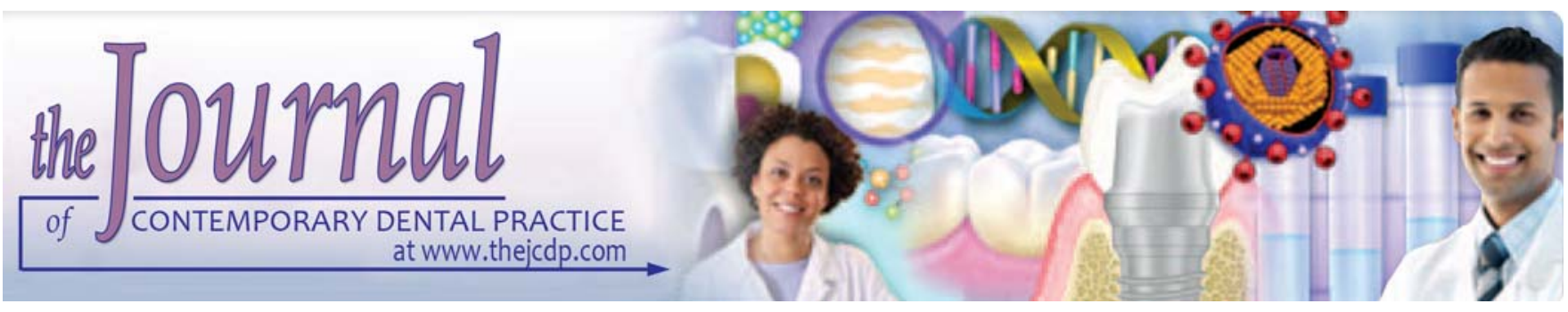

\title{
Flare-up Rate in Molars with Periapical Radiolucency in One-Visit vs Two-Visit Endodontic Treatment
}

\author{
Iftikhar Akbar, Azhar Iqbal, Mahmoud K Al-Omiri
}

\begin{abstract}
Purpose: The objective of this study was to compare postobturation flare-ups following single and two-visit endodontic treatment of molar teeth with periapical radiolucency.
\end{abstract}

Materials and methods: A total of 100 patients with asymptomatic molar teeth with periapical radiolucency were selected. They were randomly allocated into two groups. Fifty patients received complete endodontic treatment in one-visit. Fifty patients received treatment by debridement and instrumentation at the first visit followed by obturation at the second visit.

Results: $10 \%$ of patients had flare-ups in the single visit group and $8 \%$ of patients had flare-ups in the two-visit group. Number of visits did not affect the success of endodontic treatment $(p>0.05)$. Age, gender and tooth type had no effects on the occurrence of flare-ups regardless the number of visits $(p>0.05)$.

Conclusion: One-visit endodontic treatment was as successful as two-visit endodontic treatment as evaluated by rate of flareups in asymptomatic molar teeth with periapical radiolucency.

Keywords: Chronic periapical periodontitis, Endodontic flare-up, Necrotic teeth, Root canal treatment.

How to cite this article: Akbar I, Iqbal A, Al-Omiri MK. Flare-up Rate in Molars with Periapical Radiolucency in One-Visit vs TwoVisit Endodontic Treatment. J Contemp Dent Pract 2013; 14(3):414-418.

\section{Source of support: Nil}

Conflict of interest: None declared

\section{INTRODUCTION}

Endodontic treatment of asymptomatic necrotic teeth is a frequent type of therapy in endodontic practice. Postoperatively, patients should expect some discomfort associated with this treatment. ${ }^{1}$ However, there may be an acute exacerbation of symptoms commonly known as a flare-up that occurs in some patients resulting in moderate to severe postoperative pain and/or swelling. These severe symptoms upset both patient and dentist. ${ }^{2}$ Flare-up is defined as moderate to severe postoperative pain or moderate to severe swelling that begins 12 to 48 hours after treatment and lasts at least 48 hours. ${ }^{3}$ The incidence of an endodontic flare-up has been reported to be 1.4 to $16 \%$ of the time. The frequency of flare-up in necrotic pulp cases is significantly higher than vital pulp cases. ${ }^{4}$

Flare-up may occur with the best of the therapy but most flare-ups occur when improper treatment is rendered or when insufficient time is allowed for specific modalities of therapy. ${ }^{5}$

The causative factors of flare-ups comprise mechanical, chemical and/or microbial injury to the pulp or periradicular tissues. Mechanical and chemical injuries are usually associated with iatrogenic factors, such as over instrumentation, apical extrusion of irrigants and medications, perforation and canal zipping. ${ }^{6}$

Most cases of flare-ups occur as a result of acute periradicular inflammation and the intensity of inflammatory response is directly proportional to the intensity of tissue injury. Microbial injury to the periradicular tissues is probably the commonest cause of flare-ups. Although microbial insult can be coupled with iatrogenic factors, it can sometimes occur even when the root canal procedures are judicious and careful. Apical extrusion of contaminated debris to the periradicular tissues is one of the principal causes of postoperative pain. ${ }^{7}$

Traditionally root canal treatment has been divided into two or more appointments to disinfect the canals, improve patient comfort and observe healing before permanent restoration. However, one-visit endodontic treatment is well accepted by patients and prevents the recontamination of root canals between appointments. ${ }^{8}$

Considerable controversy exists over the question whether it is preferable to complete endodontic therapy in one or multiple appointments. The decision is centered mainly in two aspects. The predisposition to the flare-ups and the longterm prognosis of the teeth treated in a single appointment. ${ }^{9}$ 
This controversy practically falls to the treatment of necrotic teeth with apical injury, since they are those that are usually associated with flare-ups and endodontic failures. ${ }^{10}$ However, some studies showed that flare-ups and endodontic failures regardless the number of appointments. ${ }^{11,12}$ So, the number of appointments is not a predisposing factor for endodontic flare-ups, and that these appear at random not mattering if the teeth were treated in a single appointment.

The other aspect is the proposition of success/failure that can be obtained through endodontic treatment in one appointment. The controversy behind this could be due to the great propagation and bacterial proliferation in the root canals of necrotic teeth with apical injury. ${ }^{13}$

The defenders of the multivisit endodontic treatment in several appointments postulate that complete disinfection of the root canal in one appointment is impossible; they propose the use of intracanal calcium hydroxide to eliminate the bacteria that could not be eliminated during the biomechanical preparation. ${ }^{14}$ However, some researchers reported the use of calcium hydroxide for 7 days, and found that a complete disinfection of the canal is not possible and that a bacterial recolonization occurred at the levels similar to what was before the instrumentation of the canal. It has also been demonstrated that calcium hydroxide is difficult to remove completely from the walls of the root canal due to its clogging, and its presence can affect the quality of apical seal. ${ }^{15}$

All the teeth can be treated suitably in one appointment without concerning their pulpal and/or periapical status. But, the number of canals, the time available and the ability of the operator are among the factors that could make it difficult to accomplish the treatment in the same appointment. ${ }^{16}$

There is controversy regarding endodontic treatment in one-visit or two-visit, so the rationale of this study was to see whether any difference existed between the rates of flare-ups when doing endodontic therapy of molars with periapical radiolucency in one-visit compare to two-visit endodontic treatment.

\section{MATERIALS AND METHODS}

This cross-sectional comparative clinical study was performed on 100 asymptomatic molar teeth with periapical radiolucency. The patients were recruited from the outpatients in the Department of Conservative Dentistry, Punjab Dental Hospital, Lahore, Pakistan. The study spread over a 6 months period. The study was approved by Punjab Dental Hospital, and an informed consent was obtained from each patient before participation in the study.

The patients were randomly assigned into two groups. Involved teeth for 50 patients were treated in one-visit, and the teeth of the other 50 patients were treated in two-visit.
Diagnosis was made based on history and periapical radiograph of the involved tooth. Patients' demographic information like age, gender and address was also obtained. Standard treatment protocol for both groups included history taking, clinical examination, preoperative periapical radiograph, local anesthesia, rubber dam isolation, caries excavation and standard access preparation. The working length was determined radiographically from a coronal reference to a distance $1 \mathrm{~mm}$ short of the radiographic apex. The root canals were cleaned and shaped using the stepback technique by k files and gates Glidden drills. Canal filing was followed by irrigation of the canal with $2 \mathrm{ml}$ sodium hypochlorite (2.5\%) using a syringe with a 27 gauge needle followed by normal saline. The root canals were obturated with multiple gutta-percha cones and a zinc oxideeugenol based sealer, using the lateral condensation technique. However, for the two-visit cases, an interappointment calcium hydroxide was placed in the root canals, and the canals were obturated during a second visit. The symptoms were then recorded after obturation and then daily for 7 days.

\section{STATISTICAL ANALYSIS}

The data were analyzed using the SPSS computer software (Statistical Package for the Social Sciences, version 11.0, SPSS Inc, Chicago, IL, USA). First, simple descriptive frequency tests for the study variables were carried out and processed. Then, the associations between the variables as well as the flare-up rates in both groups were compared through Chi-square test. For all statistical analysis, the significance level was set at $\mathrm{p} \leq 0.05$.

\section{RESULTS}

One hundred patients (51 males and 49 females, age range $=12-40$ years old, mean age $=26 \pm 5$ years) were included in this study. They were divided into two groups, 50 in each group. One group was treated in one-visit and the other in two-visit.

Patients were divided into five groups according to their age; 12 to 15 years, 16 to 20 years, 21 to 25 years, 26 to 30 years, 31 to 35 years and 36 to 40 years group (Table 1 ).

\begin{tabular}{|c|c|c|c|}
\hline $\begin{array}{l}\text { Patients age } \\
\text { (years) }\end{array}$ & $\begin{array}{l}\text { Flare-up } \\
\text { (\%) }\end{array}$ & $\begin{array}{c}\text { No flare-up } \\
\text { (\%) }\end{array}$ & Total \\
\hline $12-15$ & $0(0)$ & 11 (100) & $11(100 \%)$ \\
\hline $16-20$ & $3(8.1)$ & 34 (91.9) & 37 (100\%) \\
\hline $21-25$ & 3 (15) & 17 (85) & $20(100 \%)$ \\
\hline $26-30$ & $1(6.7)$ & 13 (93.3) & $14(100 \%)$ \\
\hline $31-35$ & $1(10)$ & $8(90)$ & 9 (100\%) \\
\hline $36-40$ & 1 (14.3) & $6(85.7)$ & $7(100 \%)$ \\
\hline
\end{tabular}

Chi-square $\left(\chi^{2}\right)=2.303 ; \mathrm{df}=5 ; \mathrm{p}=0.806$ 
Flare-ups of endodontic treatment occurred in $0 \%$ of patients who aged 12 to 15 years, 3\% of each of 16 to 20 and 21 to 25 years old patients, $2 \%$ of each of 26 to 30 and 31 to 35 years old patients, and 1\% of 36 to 40 years old patients (Table 1).

Using Chi-square analysis, no significant relation was found between age and flare-up of endodontic treatment (p $=0.806)$ (Table 1$)$.

Regarding gender, only three males (5.9\%) reported flare-up while six females (12.2\%) had flare-up of the treatment they received. However, analysis using Chi-square revealed no significant relation between gender and treatment flare-up $\left(\chi^{2}=1.25, \mathrm{df}=1, \mathrm{p}=0.226\right)$.

In total, 35 maxillary and 65 mandibular molars were treated in this study. Only two maxillary molars (5.7\%) were affected by flare-up of the treatment, while seven mandibular molars (10.8\%) were reported to have flare-up. No significant differences were found between the rates of flare-up in maxillary and mandibular molars $\left(\chi^{2}=0.71, \mathrm{p}=0.399\right)$.

Out of 50 patients treated in one-visit, five (10\%) experienced a flare-up. Also, out of 50 patients treated in two-visit, four (8\%) experienced a flare-up.

Using Chi-square analysis, no significant differences in flare-up rates were found when single and two-visit techniques were used $(p=0.727)$ (Table 2).

Table 2: Distribution of endodontic flare-ups among the study population according to the number of treatment visits $(n=100)$

\begin{tabular}{lccc} 
Flare-ups & $\begin{array}{c}\text { One-visit } \\
\text { group }\end{array}$ & $\begin{array}{c}\text { Two-visit } \\
\text { group }\end{array}$ & Total \\
\hline Present & $5(10 \%)$ & $4(8 \%)$ & $9(9 \%)$ \\
Absent & $45(49.5 \%)$ & $46(50.5 \%)$ & $91(91 \%)$ \\
\hline Total & $50(100 \%)$ & $50(100 \%)$ & $100(100 \%)$ \\
\hline
\end{tabular}

Chi-square $\left(\chi^{2}\right)=0.122 ; \mathrm{df}=1 ; \mathrm{p}=0.727$

\section{DISCUSSION}

Postoperative pain and discomfort after endodontic treatment is a displeasing occasion for the patients and clinicians. Although postoperative pain associated with root canal therapy is a poor indicator of long-term success, the occurrence and the control of pain are of clinical interest in endodontics. ${ }^{17}$

Molar teeth are the most difficult teeth to manage endodontically due to limited access and canal variations, including multiple apexes and lateral canals. They are more difficult to debride thoroughly and may therefore be predisposed to postobturation complications. Molar necrotic teeth had greater rate of flare-ups than vital molar teeth. ${ }^{21}$

Comparing single and multiple-visit procedures, the question of the incidence of postoperative pain and flareups had been posed. Severe pain during endodontic therapy was a significant problem. Different studies reported various incidences of postoperative pain and flare-ups, due to differences in the criteria used in these studies. Many studies found no advantage for one-visit or two-visit treatment regarding flare-ups, postoperative symptoms or long-term healing. ${ }^{18-20}$ This study also found no advantage or disadvantage offered by one- visit or two-visit treatment regarding flare-ups. The results of this study were similar to those reported by previous studies ${ }^{18-20}$ where no significant differences in the incidence of postoperative pain were observed, when comparing single- $v$ s multiple-visit endodontic treatment.

However, the results oppose the findings of previous studies that reported higher rates of endodontic flare-up following multiple visits endodontic treatment when compared to single visit endodontics. ${ }^{11,19,21}$ Eleazer and Eleazer ${ }^{21}$ reported more flare-ups for the multiple-visit group (8\%) and (3\%) for the single visit group in necrotic molars. This was a significant finding with an advantage for one-visit treatment. Similar findings were reported by other researchers. ${ }^{11,19}$

In contrast, Oginni and Udoye ${ }^{22}$ reported more flareups in the single visit group (18.3\%) than in the multiplevisit groups (8.1\%), showing a disadvantage for single visit treatment.

In this study, age had no significant relationship with rate of endodontic flare-up. This concur the results of Oginni and Udoye $^{22}$ who found no relation between rate of flareup and age. Similar findings were also reported by Morse et $\mathrm{al}^{23}$ and Eleazer and Eleazer. ${ }^{21}$ However, Balaban et $\mathrm{al}^{24}$ treated necrotic teeth and found that there was a greater tendency to develop flare-up in patients less than 50 years old. The decrease in patient's response might be related to decreased pulp canal size, decrease blood flow to alveolus and decreased inflammatory responses in older patients.

In this study, no relationship was found between gender and rate of flare-up. This is in agreement with previous studies. ${ }^{19,21,23,25}$ On the other hand, Fox et $\mathrm{al}^{26}$ and Genet et $\mathrm{al}^{27}$ reported higher rate of endodontic flare-ups in females. This could be explained by that females tend to seek and accept treatment more willingly, as the presence of symptoms is readily perceived as indicators of disease by females. ${ }^{28}$ Furthermore; physicians believe that females suffer more commonly from psychosomatic illness and that their pain is governed by emotional factors. Another possible explanation is based on emerging evidence that biological differences between genders may explain increased pain prevalence in females. ${ }^{29}$ Also, no significant differences were found between the rates of flare-ups in maxillary and mandibular teeth. This concur the results of previous studies. ${ }^{25,26}$ The results however were contrary to the 
findings of Eleazer and Eleazer ${ }^{21}$ and Alacam ${ }^{30}$ who reported significantly higher incidence of flare-ups in mandibular teeth when compared to maxillary teeth. This could be explained on the basis that the mandible has a thick cortical plate than the upper jaw and this might cause the accumulation of exudates, which caused more pressure compared with the maxilla.

An antibacterial dressing (calcium hydroxide) was used in the two-visit group, however, it did not reduce or prevent the incidence of flare-ups. This raised the question of whether its provision offers any advantage.

In this study, five flare-ups occurred in one-visit group. Three cases were treated with nonsteroidal antiinflammatory drugs and two required obturation removal. In the two-visit group, a total of four flare-ups occurred. The two which occurred after first appointment were treated with further debridement and dressings; and further two appointments were given. The other two flare-ups occurred after obturation; these were mild and treated with nonsteroidal anti-inflammatory drugs. None of the flare-ups were accompanied by systemic signs such as fever. Therefore, none of the flare-ups posed a great difficulty in treatment, whether in one- or two-visit group.

Finally, it is important for the practitioner to have a clinical sense and adequate experience of what can be done once the rubber dam is placed and work is commenced on the tooth. Therefore; the endodontic competence of dentists becomes the overriding factor in determining the outcome of any treatment. Once a high level of competence is attained, one-visit endodontics can be successfully performed if clinicians choose their cases carefully and adhere to basic endodontic principles. ${ }^{21}$

\section{CONCLUSION}

One-visit endodontic treatment was as successful as twovisit endodontics in asymptomatic molar teeth with periapical radiolucency. Postoperative flare-ups had no relationship with number of treatment visits, patients' age, gender or tooth type.

\section{REFERENCES}

1. Ghoddusi J, Javidi M, Zarrabi MH, Bagheri H. Flare-ups incidence and severity after using calcium hydroxide as intracanal dressing. NY State Dent J 2006;72:24-28.

2. Mahmood S, Yaha AA, Saad HA, Hussain KA. Reasons for root canal treatment in students and interns clinics in college of dentistry, King Saud University, Saudi Arabia. J Pak Dent Assoc 2003;12:33-36.

3. Pickenpaugh L, Reader A, Beck M, Meyers WJ, Peterson LJ. Effect of prophylactic amoxicillin on endodontic flare-up in asymptomatic, necrotic teeth. J Endod 2001;27:53-56.
4. Inamoto K, Kojima K, Hamguchi A, Nakata K, Nakamura H. A survey of the incidence of single-visit endodontics. Endod J 2002;28:371-377.

5. Jose F, Siqueria JF Jr, Isabela N, Faveiri A, Andreia G, Sergio $\mathrm{M}$, et al. Incidence of postoperative pain after intra canal procedures based on an antimicrobial strategy. J Endod 2002;28:457-460.

6. Seltzer S, Naidorf IJ. Flare-ups in endodontics: Part 1. Etiological factors. J Endod 2004;30:476-481.

7. Kvist T, Molander A, Dahlen G, Reit C. Microbial evaluation of one and two visits endodontic treatment of teeth with apical periodontitis. J Endod 2004;30:572-576.

8. Sathorn C, Parushus P, Messer HH. Effectiveness of single versus multiple-visit endodontic treatment of teeth with apical periodontitis: A systemic review and meta-analysis. Int Endod J 2005;38:347-355.

9. Walton RE. Interappointment flare-ups: incidence, related factors, prevention, and management. Endodontic Topics 2002;3:67-77.

10. Sjogren U, Figdor D, Persson S, Sundqvist G. Influence of infection at the time of root canal filling on the outcome of endodontic treatment of teeth with apical periodontitis. Int Endod J 1997;30:297-306.

11. Trope M. Flare-up rate of single visit endodontics. Int Endod J 1991;24:24-27.

12. Trope M, Delano OR, Ostavik D. Endodontic treatment of teeth with apical periodontitis: single versus multivisit treatment. J Endod 1999;25:345-350.

13. Caliskan M, Sen B. Endodontic treatment of teeth with apical periodontitis using calcium hydroxide: long term study. Endod Dent Traumatol 1996;12:215-221.

14. Sjogren U, Hagglund B, Sundqvist G, Wing K. Factors affecting the long term results of endodontic treatment. J Endod 1990;16:498-504.

15. Peters LS, Winkelhoff A, Buijs J, Wesselink P. Effect of instrumentation, irrigation and dressing with periapical calcium hydroxide on infection in pulp less teeth with bone lesions. Int Endod J 2002;35:13-26.

16. Spagberg LS. Evidence-based endodontics: the one-visit treatment idea. Oral Surg Oral Med Oral Path 2001;91:617-618.

17. Al-Negrish AR, Habaheh R. Flare-up rate related to root canal treatment of asymptomatic pulpally necrotic central incisor teeth in patients attending a military hospital. J Dent 2006;34:635-640.

18. Oliet S. Single-visit endodontics. A clinical study. J Endod 1983;9:147-152.

19. DiRenzo A, Gresla T, Johnson B, Rogers M. Tucker D, BeGole E. Postoperative pain after 1- and 2-visit root canal therapy. Oral Surg Oral Med Oral Pathol Oral Radiol Endod 2002;93:605-610.

20. Soltanoff W. A comparative study of single visit and multivisit procedures. J Endod 1978;4:278-281.

21. Eleazer P. Eleazer K. Flare-up rate in pulpally necrotic molars in one-visit versus two-visit endodontic treatment. J Endod 1998;24:614-616.

22. Oginni AO, Udoye CI. Endoddontic flare-ups: comparison of incidence between single and multivisit procedures in patients attending a Nigerian teaching hospital. BMC Oral health 2004;4:4.

23. Morse KD, Koren LZ, Esposite JV. Infection flare-ups and their prevention. Int J Psychosm 1986;33:2-3.

24. Balaban FS, Skidmore AE, Griffin JA. Acute exacerbations following treatment of necrotic pulps. J Endod 1984;10:78-81. 
25. Walton R, Fouad A. Endodontic inter-appointment flare-ups: a prospective study of incidence and related factors. J Endod 1992;18:172-177.

26. Fox J, Atkinson J, Dinin P. Incidence of pain following onevisit endodontic treatment. Oral Surg 1970;30:123-130.

27. Genet IM, Wesselink PR, Thoden Van Velzen SK. Preoperative and operative factors associated with pain after the first endodontic visit. Int J Endod 1987;20:53-64.

28. Molander A, Warfvinge J, Reit C, Kvist T. Clinical and radiographic evaluation of one and two visit endodontic treatment of asymptomatic teeth with apical periodontitis. A randomized clinical trial. J Eondod 2007;33:1145-1148.

29. Colemeco S, Becker LA, Simpson M. Sex bias in the assessment of patient complaints. J Fam Pract 1983;16:117-121.

30. Alacam T, Tinaz AC. Inter-appointment emergencies in teeth with necrotic pulps. J Endod 2002;28:375-377.

\section{ABOUT THE AUTHORS}

\section{Iftikhar Akbar}

Assistant Professor, Department of Conservative Dentistry, Faculty of Dentistry, Al-Jouf University, Sakaka, Saudi Arabia

\section{Azhar lqbal}

Assistant Professor, Department of Conservative Dentistry, Faculty of Dentistry, Al-Jouf University, Sakaka, Saudi Arabia

\section{Mahmoud K Al-Omiri (Corresponding Author)}

Professor and Senior Consultant, Department of Prosthodontics Faculty of Dentistry, University of Jordan, Amman-11942, Jordan e-mail: alomirim@yahoo.co.uk 